\title{
Monitoring and Diagnosis of Depression from Daily Motor Activity
}

\author{
Ivy Y. Xie \\ Newark Academy, Livingston, NJ, USA
}

\begin{abstract}
Depression is one of the most common mental disorder associated with suicide. Timely diagnosis and intervention of depression will improve life quality and reduce suicidal rate. Recent studies have shown that motor activities measured from a wearable sensor may correlate with the depression state. I develop a machine learning model to diagnose depression using motor activity data. My model improves the base-line performance by 44\%, suggesting the potential of artificial intelligence in mental health management.
\end{abstract}

\section{Introduction}

Depression is one of the most common mental disorder associated with suicidal behavior. Conventionally, the monitoring and diagnosis of depression rely on self-assessment questionnaires by users and/or phone/in-site interviews by psychologists. The requirement of user interactions often delays the detection of changes in the depression state. The recent development of wearable mobile sensors provides new opportunities for diagnosing depression without the need for user interaction. In this paper, my objective of the study is to develop accurate machine learning models for the diagnosis of the depression using mobile sensor data[1]. My model significantly outperforms base-line models, suggesting the potential of machine learning in the monitoring, diagnosis, and prevention of mental disorders. My ultimate goal is to develop a wearable device coupled with artificial intelligence for mental health management.

\section{Methods}

Training and testing data were from Depresjon database (https://datasets.simula.no/depresjon/). The dataset contains multiple days ( 15 days on an average) of motor activity recordings of 23 depressed patients and 32 healthy controls using Actigraph watch. The activity was recorded every minute. I preprocessed the data as follows. First, I used the daily activity data of each individual as training and testing samples. I treated the weekday as a category variable. Second, I imputed the missing data by averaging the activity value in the same time-point for the corresponding individual. Finally, I applied the moving average smoothing to reduce the dimensionality and noisiness of the feature. After the preprocessing, I randomly drew $10 \%$ of individuals as hold-out data for the validation. I used the remaining $90 \%$ of individuals for model training and hyperparameter tuning. I applied Random Forest (RF) implemented in scikit-learn package (https://scikit-learn.org/) to train a classification model to classify the depression versus the nondepression. The reason to choose RF is that it can handle high-dimensional and categorical data. I applied the grid search to find the optimal parameters and used the hold-out individuals to measure the final performance. The metrics are precision, recall, F1 score, and Matthews correlation coefficient (MCC).

\section{Results}

Moving average smoothing was applied to address the high-dimensionality (1,441 dimensions) and noisiness in the motor activity data. Figure 1 shows the weighted F1 score for different window size. The best performance was achieved when the window size is 40 minutes. The new model (window size $=40 \mathrm{~min}$.) significantly outperforms published models[1], as shown in Table 1. The weighted F1 and MCC improve approximately $13 \%$ and $44 \%$, respectively. Even without window smoothing, the new model still performs better than the published ones. The performance improvement may attribute to the use of daily activity and the application of individualized imputation. The feature importance ranking does not show significantly important features (Figure 2). In general, the morning and afternoon activity has more predictive power than the evening activities. The night activity is the least informative.

\section{Discussion}

In this study, I develop a new machine learning model that significantly improves both the sensitivity and specificity of depression diagnosis based on the mobile sensor data. Methodologically, I show that individualized imputation and data smoothing is crucial for model performance. In terms of biomedical application, my study demonstrates the potential of artificial intelligent in mental health management. The early detection and continuous 7/24 monitoring of depression state will no doubt facilitate the intervention and treatment of depression, thereby reducing the suicidal rate and improving an individual's quality of life. In the future, I will further improve the model performance by balancing the data and testing other advanced machine learning techniques such as Gradient Boosting Tree. 


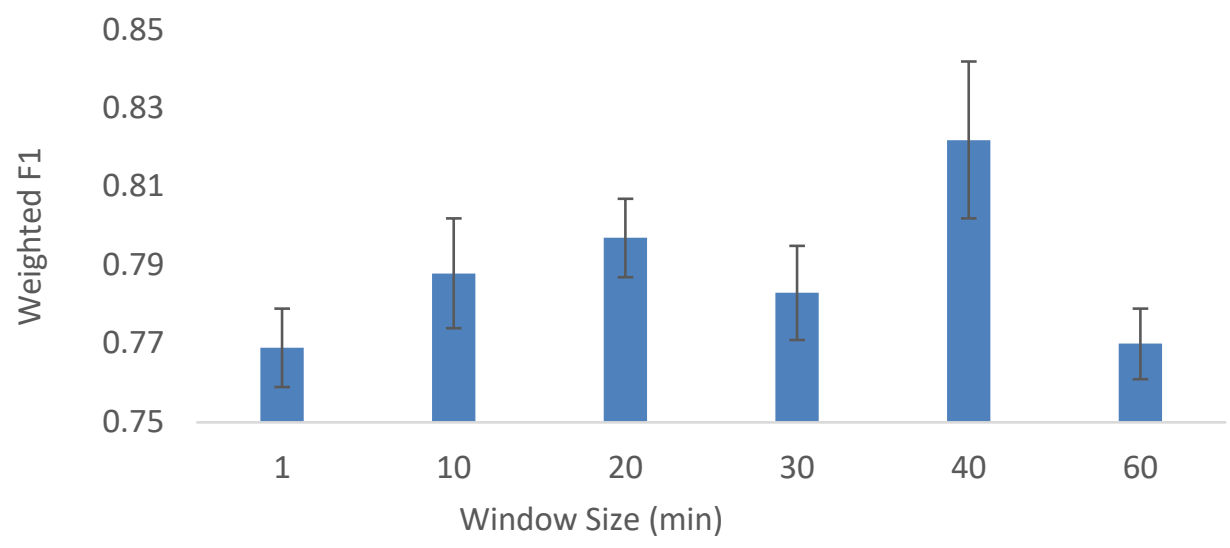

Figure 1. Weighted F1 score of Random Forest model when using features with different window sizes.

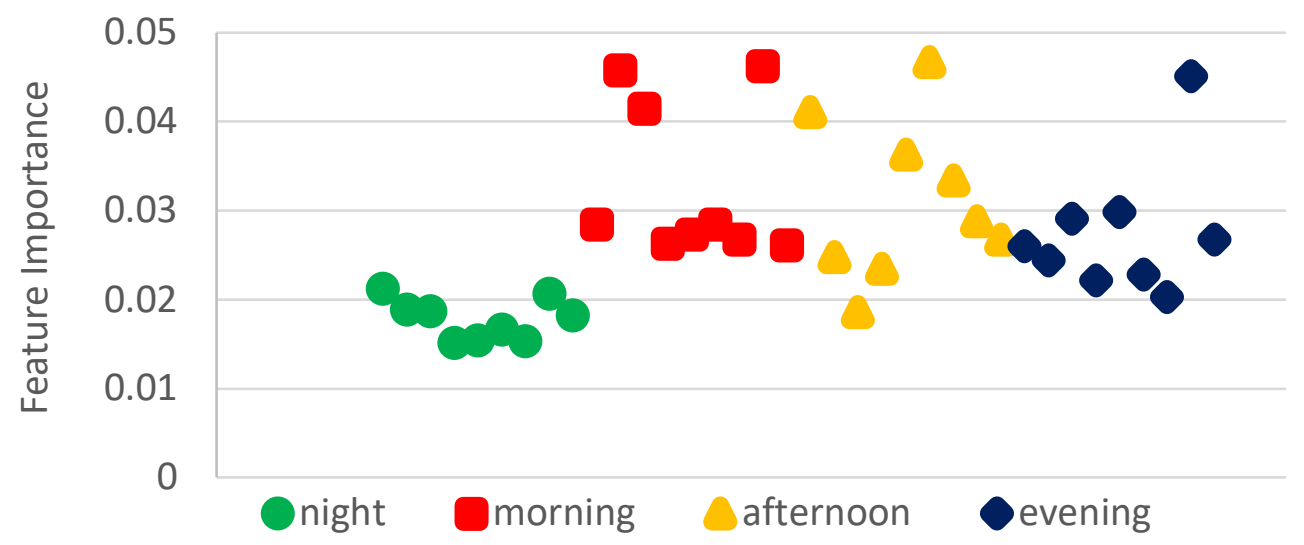

Figure 2. Feature importance

Table 1. Performance comparison between my model and the best-published model ${ }^{1}$

\begin{tabular}{|l|l|l|l|l|c|}
\hline Classifier & Class & Precision & Recall & F1 & MCC \\
\hline \multirow{3}{*}{ My model } & Depressed & $0.754+/-0.029$ & $0.777+/-0.042$ & $0.765+/-0.028$ & - \\
\cline { 2 - 6 } & Non-depressed & $0.865+/-0.022$ & $0.848+/-0.023$ & $0.856+/-0.016$ & - \\
\cline { 2 - 6 } & Weighted & $\mathbf{0 . 8 2 4 + / - 0 . 0 2 0}$ & $\mathbf{0 . 8 2 1 + / - 0 . 0 2 0}$ & $\mathbf{0 . 8 2 2 + / - 0 . 0 2 0}$ & $\mathbf{0 . 6 2 2 + / - 0 . 0 4 3}$ \\
\hline \multirow{2}{*}{$\begin{array}{l}\text { The best- } \\
\text { published } \\
\text { model }^{1}\end{array}$} & Depressed & 0.577 & 0.721 & 0.638 & - \\
\cline { 2 - 6 } & Non-depressed & 0.836 & 0.734 & 0.780 & - \\
\cline { 2 - 6 } & Weighted & 0.735 & 0.727 & 0.724 & 0.433 \\
\hline
\end{tabular}

\section{References}

1. Garcia-Ceja E, Riegler M, Jakobsen P, Torresen J, Nordgreen T, Oedegaard KJ, Fasmer OB. Depresjon: a motor activity database of depression episodes in unipolar and bipolar patients. MMsys' 18 Proceedings of the $9^{\text {th }}$ ACM Multimedia Conference. 2018;pp472-477. 